Revista de

Contabilidade e

Organizações

www.rco.usp.br
DOI: http://dx.doi.org/10.11606/issn.1982-6486.rco.2020.164058

Journal of

Accounting and

Organizations

\title{
Influência de fatores contingenciais no desempenho municipal: evidências inferenciais
}

\section{Influence of contingency factors on municipal performance: inferential evidence}

Filipy Furtado Sell ${ }^{\mathrm{a}}$, Ilse Maria Beuren ${ }^{\mathrm{b}}$, Carlos Eduardo Facin Lavarda ${ }^{\mathrm{b}}$

aniversidade Federal do Pará

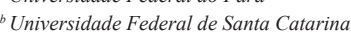

Palavras-chave

Teoria da Contingência.

Fatores contingenciais.

Desempenho municipal.
Keywords

Contingency Theory.

Contingency factors.

Municipal performance.

\section{Resumo}

Este estudo analisa a influência de fatores contingenciais no desempenho de municípios ao longo de três mandatos governamentais. Consideram-se fatores contingenciais internos (liderança organizacional, estrutura e porte organizacional) e externo (ambiente). Os dados de 293 municípios, com 3.516 observações, relativos aos três últimos mandatos encerrados, foram analisados pela regressão de dados em painel. Os resultados revelaram influência significativa de fatores contingenciais no desempenho dos municípios. Os fatores ambiente (IDHM), liderança organizacional (idade do prefeito), estrutura (despesa de pessoal per capita) e porte organizacional (densidade populacional) influenciaram positivamente o desempenho municipal (receita corrente líquida per capita). A relação positiva e significativa entre estrutura e desempenho municipal indica que o corte de gastos com pessoal nos municípios estudados não aumentará seu desempenho. Para as variáveis de controle, destaca-se que nos municípios em que o partido do prefeito é o mesmo do governador há maior possibilidade de obtenção de recursos. As demais variáveis de controle atreladas às características do prefeito não obtiveram significância estatística. Assim, a reeleição do prefeito e sua direção partidária não influenciaram no desempenho municipal.
Informações do artigo

Recebido: 12 de novembro de 2019

Aprovado: 17 de abril de 2020

Publicado: 08 de maio de 2020

\begin{abstract}
This study analyzes the influence of contingency factors on municipal performance throughout three government mandates. It considers external (environment) and internal (organizational leadership, organizational structure and size) contingency factors. Data from 293 municipalities, with 3,516 observations, related to the last three complete mandates, and were analyzed through data regression panel. The results revealed significant influence of contingency factors on the performance of the municipalities. The factors of environment (MHDI), organizational leadership (mayor's age), structure (per capita staff expenditure) and organizational size (population density) positively influenced municipal performance (net per capita income). The positive and significant relationship between structure and municipal performance indicates that the cut in personnel expenses in the municipalities will not increase their performance. For the control variables, it is noteworthy that in the municipalities where the mayor's party is the same as the governor's party, there is a greater possibility of obtaining resources. The other control variables linked to the mayor's characteristics did not obtain statistical significance. Thus, the re-election of the mayor and his party leadership did not influence municipal performance.
\end{abstract}

\section{Implicações práticas}

$\mathrm{O}$ artigo busca auxiliar a gestão pública na figura dos servidores, gestores e consultores públicos, ao evidenciar fatores contingenciais que afetam a estrutura, o comportamento organizacional e o desempenho de municípios, com vistas para melhorar a eficiência da gestão.

Copyright (C) 2020 FEA-RP/USP. Todos os direitos reservados 


\section{INTRODUÇÃO}

A avaliação de desempenho no setor público é parte integrante da proposta da nova gestão pública, visto a orientação para utilização de mecanismos gerenciais e indicadores de desempenho para a gestão eficiente dos recursos públicos (Hood, 1991; Gonzaga, Frezatti, Ckagnazaroff \& Suzart, 2016). Bezerra e Carvalho (2018) afirmam que a gestão pública brasileira passa por mudanças de práticas que direcionam o país para novas correntes teóricas que focalizam eficiência e transparência.

No entanto, as organizações governamentais, além de gerir os recursos de forma eficiente e transparente, devem avaliar e monitorar suas ações (Ramos \& Schubbach, 2012). Verbeeten (2008) aponta que a avaliação de desempenho opera como fator de aprendizagem para melhorar o desempenho e suporte para a prestação de contas. Um contraponto à avaliação de desempenho é a possibilidade de um aumento da burocracia interna sufocar a inovação e a subotimização das métricas de desempenho (Castaldelli \& Aquino, 2011).

Johnsen (2005) já indicava que o crescimento do uso de indicadores de desempenho no setor público auxilia na tomada de decisões relacionadas às questões administrativas e políticas. Apesar do crescimento do uso de indicadores de desempenho, não há um framework dedicado à mensuração do desempenho em organizações governamentais e o indicativo de quais as consequências da adoção de grupos de determinados indicadores em detrimento de outros (Boyne, Meier, O'Toole \& Walker, 2005). Ainda não há consenso sobre o tema (Boyne et al., 2005), seja em âmbito internacional ou nacional.

Nesta pesquisa fomenta-se a discussão de fatores contingenciais relacionados à avaliação de desempenho no setor público. Donaldson (2001) orienta que fatores contingenciais afetam a relação entre características e desempenho da organização. Marques, Souza e Silva (2015) destacam que fatores contingenciais compreendem elementos internos e externos à organização, que podem interferir em sua estrutura organizacional. Porporato (2011) sugere que o alinhamento de fatores contingenciais e controles gerenciais de uma organização resultará no alcance do desempenho desejado. Ressalta, no entanto, que definições universais são ineficazes, visto não haver um padrão de fatores contingenciais que afetam as organizações.

Estudos feitos no contexto brasileiro sob a lente da Teoria da Contingência utilizaram elementos diversos para capturar os fatores contingenciais, como por exemplo: ambiente e estrutura organizacional mecânica e orgânica (Fagundes, Petri, Lavarda, Rodrigues, Lavarda \& Soller, 2010); ambiente, tecnologia, estrutura, estratégia e porte organizacional (Beuren \& Fiorentin, 2014); ambiente e práticas gerenciais (Gonzaga et al., 2016); e ambiente externo, tamanho, estratégia, estrutura, tecnologia, cultura organizacional e liderança (Oliveira \& Callado, 2018). Nota-se que não há consenso quanto aos elementos internos, mas sim quanto ao elemento externo ambiente.

Neste contexto de identificação de indicadores para avaliar o desempenho no setor público brasileiro, Avellaneda e Gomes $(2015 ; 2017)$ analisaram fatores internos que podem afetar o desempenho municipal. Em 2015 os autores analisaram o efeito do tamanho no desempenho municipal e em 2017 a capacidade gerencial dos prefeitos no desempenho municipal. Avellaneda e Gomes $(2015 ; 2017)$ utilizaram o valor arrecadado no Imposto sobre a Propriedade Predial e Territorial Urbana (IPTU) como variável de desempenho e não abordaram, de forma declarada, os fatores internos suportados pela Teoria Contingencial.

Nesta perspectiva tem-se a seguinte questão de pesquisa: Qual a influência de fatores contingenciais no desempenho de municípios? Assim, o objetivo da pesquisa é analisar a influência de fatores contingenciais (ambiente, liderança organizacional, estrutura e porte organizacional) no desempenho (Receita Corrente Líquida) de municípios, ao longo de três mandatos governamentais (2005 a 2016).

Esta pesquisa visa contribuir para a literatura dedicada à análise de fatores que afetam o desempenho no setor público (ex.: Holzer et al., 2009; Drew, Kortt \& Dollery, 2014; Jung \& Kim, 2014; Avellaneda \& Gomes, 2017; Goeminne \& George, 2018; Park, 2019) e à nova gestão pública (ex.: Lapsley, 1999; Parker \& Gould, 1999; Christensen \& Yoshimi, 2003; Van der Hoek, 2005; Van de Walle \& Hammershmid, 2011; George, Van de Walle \& Hammerschmid, 2019), por discutir elementos que podem afetar o desempenho de organizações governamentais.

Diante de evidências da literatura que fatores contingenciais podem afetar as características e o desempenho da organização, esta pesquisa avança na discussão sobre fatores que podem afetar o desempenho municipal. Em especial, avança ao incluir no modelo o fator contingencial externo ambiente e ao aferir o desempenho municipal com base na Receita Correte Líquida (RCL), que se caracteriza como receita corrente que efetivamente pertence ao município, parâmetro não considerado em estudos anteriores.

Contribui também para a literatura pela análise longitudinal de fatores que afetam o desempenho municipal. De acordo com Adhikari, Derashid e Zhang (2006), estudos com essas características conseguem mitigar resultados tendenciosos pelo efeito do tempo. Hedeker e Gibbons (2006) orientam que análises longitudinais são fundamentais para inferências entre variáveis. Fávero e Belfiore (2017) argumentam que em contraponto a análises individuais, os estudos longitudinais mitigam inconvenientes na análise estatística, pois há maior variabilidade dos dados. 
Além da contribuição para avanços na literatura, esta pesquisa apresenta contribuições práticas por evidenciar fatores internos e externos às organizações governamentais que podem afetar o seu desempenho. No cenário contemporâneo brasileiro de incertezas políticas e econômicas, evidenciar fatores que possam afetar o desempenho de organizações públicas, no caso municípios, importa para a sociedade civil, servidores, gestores e órgãos de controle (Lima \& Aquino, 2019). A escolha dos municípios pesquisados é ilustrativa, e espera-se que os indicadores selecionados possam ser úteis para outras pesquisas.

\section{FATORES CONTINGENCIAIS E DESEMPENHO NO SETOR PÚBLICO}

A Teoria da Contingência prevê que fatores contingenciais influenciam nas características e no desempenho das organizações (Donaldson, 2001). Greenwood e Hinings (1976) destacam que fatores contingenciais estão implicados no grau em que a organização assegura ajustes em controles e estrutura, decorrentes de circunstâncias situacionais, para aumentar desempenho organizacional. Brignall e Modell (2000) afirmam que o princípio fundamental da Teoria da Contingência é o esforço das organizações para maximizar o desempenho e eficiência com ajustes entre ambiente e estrutura.

Teisman e Klijn (2008) ressaltam que a absorção das pressões internas e externas à organização impactam a estrutura e o comportamento organizacional. Os fatores contingenciais compreendem fatores internos e externos à organização (Wadongo \& Abdel-Kader, 2014). Além da eficiência, desempenho e comportamento organizacional, Woods (2009) destaca que influencia o desenho e a implementação do sistema de controle por meio dos fatores contingenciais como ambiente, tecnologia, estrutura, tamanho e estratégia. Donaldson (2001) classifica fatores contingenciais como qualquer variável que tenha o poder de alterar a relação entre estrutura organizacional e seu desempenho.

A literatura aplicada ao setor privado e ao setor público identifica como fatores contingenciais internos: tecnologia, estrutura, estratégia, porte organizacional, cultura organizacional e liderança; e como fator contingencial externo o ambiente (Greenwood \& Hinings, 1976; Woods, 2009; Fagundes et al., 2010; Beuren \& Fiorentin, 2014; Wadongo \& Abdel-Kader, 2014; Gonzaga et al., 2016; Oliveira \& Callado, 2018). Segundo Wadongo e AbdelKader (2014), o ambiente é um fator contingencial relevante pela incerteza ou dificuldade de previsão, dinamismo e adversidades enfrentadas pelas organizações.

Apesar da literatura aplicada aos setores privado e público coincidir na caracterização de fatores contingenciais internos e externos, Woods (2009) afirma que possuem contextos diferentes. Explica que no contexto organizacional privado prima-se pelas variáveis contingenciais de impacto financeiro, já no contexto organizacional público focaliza-se o impacto na prestação dos serviços. No entanto, o setor público brasileiro passa por mudanças alinhadas à Nova Gestão Pública (New Public Management) (Hood, 1991), que traz para as organizações públicas características do setor privado, como a implantação de sistemas de controle gerencial, foco no desempenho, mudanças na prestação dos serviços públicos e nas características dos gestores (Oliveira, Sant'Anna \& Vaz, 2010).

Christensen e Yoshimi (2003) observam em sua pesquisa que a Nova Gestão Pública não explica completamente os caminhos percorridos para o desenvolvimento dos relatórios de desempenho. Destacam que alguns pontos de vista adicionais são necessários para compreender o fenômeno estudado, dentre eles a Teoria Contingencial. Outra evidência de autores que recorreram à Teoria Contingencial para explicar o fenômeno estudado é de George, Van de Valle e Hammerschmid (2019), ao argumentarem, com base em Donaldson (2001), que municípios maiores e com objetivos claros são mais propensos a utilizar mecanismos de gestão devido ao contexto organizacional.

Infere-se do exposto que a literatura destinada a verificar fatores que influenciam o desempenho municipal, como os estudos de Avellaneda e Gomes $(2015$; 2017) que verificaram a influência do tamanho do município e das características do prefeito, observaram efeitos de fatores internos no desempenho. Assim avança-se ao analisar fatores externos e internos no desempenho municipal. Esta agenda de pesquisa mostra-se prolífera, visto estar presente em estudos publicados desde 1976 (ex.: Greenwood \& Hinings, 1976) até recentes (ex.: George, Van de Valle \& Hammerschmid, 2019). 


\section{METODOLOGIA}

Para analisar a influência dos fatores contingenciais ambiente, liderança organizacional, estrutura e porte organizacional no desempenho municipal, a população da pesquisa compreendeu os 295 municípios que compõem o estado de Santa Catarina. Os municípios de Balneário Rincão (fundado em 2003) e de Pescaria Brava (fundado em 2013) foram excluídos da pesquisa devido à falta de dados relativos ao período de 2005 a 2011. Assim, a pesquisa abarcou 293 municípios, com 3.516 observações, cuja escolha é ilustrativa.

O período analisado de 2005 a 2016 compreende três mandatos governamentais completos (12 anos). Optou-se por utilizar os últimos três mandatos completos para mitigar vieses na interpretação dos dados face ao uso de dados orçamentários e características dos prefeitos municipais. Os dados dos municípios catarinenses foram coletados nos seguintes sites: Tribunal de Contas do Estado de Santa Catarina, Federação das Indústrias do Estado do Rio de Janeiro, Tribunal Superior Eleitoral, Instituto Brasileiro de Geografia e Estatística e portais de transparências dos municípios.

Na Tabela 1, apresentam-se as variáveis dependentes, independentes e de controle.

Tabela 1. Variáveis pesquisadas

\begin{tabular}{|c|c|c|}
\hline Variável & Medida & Autor \\
\hline \multicolumn{3}{|c|}{ Variável dependente } \\
\hline Receita Corrente Líquida per capita & $R C L p c=R C L /$ População & Autores da pesquisa \\
\hline \multicolumn{3}{|c|}{ Variável independente } \\
\hline Ambiente & $\begin{array}{l}\text { Índice de Desenvolvimento Humano Municipal } \\
\text { (IDHM) }\end{array}$ & Autores da pesquisa \\
\hline
\end{tabular}

Capacidade do Prefeito referente a Idade

(IP) $=$ Log Idade do Prefeito

Capacidade do Prefeito referente a Escolaridade

$(\mathrm{EP})=1$.Mal consegue ler seu próprio nome

2.Ensino Fundamental Incompleto

Liderança Organizacional

3.Ensino Primário

4.Segundo Grau Incompleto

5.Segundo Grau Completo

6.Graduação Incompleta

7.Graduação Completa

Avellaneda e Gomes

8.Pós-Graduação

\begin{tabular}{|c|c|c|}
\hline Estrutura & $\begin{array}{c}\text { Despesa de Pessoal per capita } \\
(D P p c)=D P / \text { População }\end{array}$ & \\
\hline \multirow{2}{*}{ Porte Organizacional } & $\begin{aligned} & \text { Densidade Populacional } \\
(\text { Dens } P)= & \text { População/Área do município }\left(\mathrm{km}^{2}\right)\end{aligned}$ & \\
\hline & $L P=\log$ População & \\
\hline \multicolumn{3}{|c|}{ Variável de controle } \\
\hline $\begin{array}{l}\text { Alinhamento partidário entre } \\
\text { Governo e Prefeito }\end{array}$ & $\begin{array}{l}\text { Governo e Prefeito são do mesmo partido político? } \\
\qquad 1 \text { = Sim e } 2 \text { = Não }\end{array}$ & \multirow{4}{*}{$\begin{array}{c}\text { Avellaneda e Gomes } \\
\text { (2015) }\end{array}$} \\
\hline Ex-prefeito & $\begin{array}{l}\text { Prefeito possui eleição anterior? } \\
1 \text { = Sim e } 2 \text { = Não }\end{array}$ & \\
\hline Direção Partidária & $\begin{array}{l}\text { Qual a direção partidária do Prefeito? } \\
1 \text { = Esquerda, } 2 \text { = Direita ou } 3=\text { Centro }\end{array}$ & \\
\hline PIB per capita & PIBpc $=$ PIB / População & \\
\hline
\end{tabular}

Fonte:elaborada pelos autores.

A escolha da variável dependente Receita Corrente Líquida, para avaliar o desempenho municipal, decorre da perspectiva de que ela resulta nos valores de arrecadação corrente que efetivamente ficam para o município (para composição ver art. $2^{\circ}$ da Lei Complementar no 101/2000, Lei de Responsabilidade Fiscal). A RCL é um relevante indicador das finanças públicas e métrica de gestão financeira para o atendimento de metas estabelecidas na Lei de Responsabilidade Fiscal (TCU, 2015). O desempenho da RCL (maior ou menor montante de RCL disponível para o gestor municipal) infere o planejamento dos municípios e demanda destreza do gestor municipal para a aplicação dos recursos em benefício da sociedade e cumprimento das metas da Lei de Responsabilidade Fiscal. 
As variáveis independentes compreendem fatores internos e externos à organização, conforme preconiza a Teoria da Contingência. Como variável externa, denominada ambiente, utilizou-se o Índice de Desenvolvimento Humano Municipal estimado pela Federação das Indústrias do Estado do Rio de Janeiro. A escolha desse índice deu-se por compreender em seu cálculo dados relacionados ao emprego, renda, saúde e educação, independentes, ou não, da ação do município. Além dessa variável capturar o ambiente externo à organização tem-se para este indicador e, também, para a Receita Corrente Líquida, as seguintes propriedades requeridas para um indicador: relevância, confiabilidade na medida, factibilidade operacional para sua obtenção, periodicidade, comparabilidade da série histórica, especificidade, validade de representação e transparência metodológica em sua construção (Gonzaga et al., 2016).

Quanto ao ambiente interno, definiram-se as variáveis liderança organizacional, estrutura e porte organizacional, seguindo a pesquisa de Avellaneda e Gomes (2015) e a chamada de Greenwood e Hinings (1976), de que pesquisas em organizações públicas devem considerar a existência de representantes eleitos. O mesmo deuse para as variáveis de controle. Ressalta-se que Avellaneda e Gomes (2017) não utilizaram a Teoria Contingencial como lente teórica para verificar a influência da qualidade do prefeito no desempenho municipal, mas apresentaram indícios suportados pelos preceitos dessa Teoria.

Na variável de controle Direção Partidária utilizou-se a perspectiva de Carreirão (2006), ao classificar os partidos políticos brasileiros em: Direita (DEM, PR, PP, PFL, PRN, PDC, PL, PTB, PSC, PSP, PRP, PSL, PSD e PRONA, além do PMDB e PSDB, mesmo tendo características centrais, pela orientação conservadora considerouse como partido de direita); Esquerda (PT, PDT, PPS, PCdoB, PSB, PV, PSTU, PCO e PMN); e Centro (PHS, PPS, PRB, PRTB, PSD, PSDC, PT DO B, PTC e PTN).

Face às variáveis apresentadas e a natureza transversal dos dados da variável dependente, estimou-se o modelo pelos mínimos quadrados ordinários. Assim, segue o modelo:

$$
R C L_{p c_{i}}=\propto+\beta_{1}(A m b)+\beta_{2}(I P)+\beta_{3}(E P)+\beta_{4}\left(D P_{p c}\right)+\beta_{5}(D e n s P)+\beta_{6}(L P)+\beta_{7}\left(X_{i}\right)+\beta_{8} e
$$

Onde, " $X_{i}$ " refere-se às variáveis de controle e " $e$ " é o termo de erro.

$\mathrm{Na}$ execução do modelo utilizou-se a abordagem da regressão para dados em painel. Para verificar a presença de heterocedasticidade aplicou-se o teste de Poi e Wiggins (2001) e o teste de Wald, já que o melhor estimador é Efeitos Fixos (pelos testes F de Chow e Hausman); e para a autocorrelação aplicou-se o teste de Wooldrige (Fávero \& Belfiore, 2017). Constatou-se que há presença de heterocedasticidade e autocorrelação nos dados.

Gujarati e Porter (2011) já orientavam que é factível e corriqueiro que se tenha problemas de heterocedasticidade e autocorrelação em dados em painel. Uma alternativa é reestimar o modelo, utilizando os estimadores dos mínimos quadrados generalizados factíveis (Gujarati \& Porter, 2011; Fávero \& Belfiore, 2017). Assim, o modelo foi reestimado com os estimadores mínimos quadrados generalizados factíveis com autocorrelação, controlada pelo método de Durbin-Watson. O modelo foi executado no programa Stata ${ }$.

\section{DESCRIÇÃO E ANÁLISE DOS RESULTADOS}

Nesta seção, inicialmente apresentam-se as estatísticas descritivas, após a matriz de correlação e, por fim, o resultado da regressão de dados em painel do modelo proposto. Na Tabela 2 apresentam-se as estatísticas descritivas dos dados. 
Tabela 2. Estatística descritiva

\begin{tabular}{lcccc}
\hline \multicolumn{1}{c}{ Variável } & Mínimo & Máximo & Média & Desvio Padrão \\
\hline 1. Receita Corrente Líquida per capita & 244,35 & $9.642,71$ & $2.241,30$ & $1.162,93$ \\
2. Ambiente & 0,4284 & 0,928 & 0,7211 & 0,0758 \\
3a. Idade do Prefeito & 24 & 80 & 49,44 & 8,46 \\
3b. Escolaridade do Prefeito & 1 & 8 & 5,43 & 1,75 \\
4. Despesa de Pessoal per capita & 69,13 & $4.660,70$ & $1.088,63$ & 595,98 \\
5a. Densidade Populacional & 1,965 & $2.848,76$ & 83,95 & 195,32 \\
5b. Logarítimo da População & 7,20 & 13,25 & 9,09 & 1,40 \\
6. Alinhamento Partidário & 1 & 2 & 1,7647 & 0,4241 \\
7. Ex-Prefeito & 1 & 2 & 1,7050 & 0,4560 \\
8. Direção Partidária & 1 & 3 & 1,9203 & 0,4403 \\
9. Produto Interno Bruto per capita & 1,42 & 166,37 & 20,11 & 11,48 \\
\hline
\end{tabular}

Fonte:elaborada pelos autores.

A estatística descritiva evidencia os valores mínimo, máximo, média e desvio padrão dos dados coletados. Na Tabela 3 evidenciam-se os municípios e o respectivo ano destacados com os valores mínimo, máximo e média das variáveis dependente e independentes.

Tabela 3. Apresentação dos municípios destacados na estatística descritiva

\begin{tabular}{|c|c|c|c|c|}
\hline & Variável & Mínimo & Máximo & Média \\
\hline Dependente & $\begin{array}{l}\text { 1. Receita Corrente } \\
\text { Líquida per capita }\end{array}$ & Brunópolis (2010) & $\begin{array}{l}\text { Bom Retiro } \\
\quad(2016)\end{array}$ & $\begin{array}{c}\text { José Boiteux (2013) } \\
\text { Campo Alegre (2014) } \\
\text { Itaiópolis (2016) }\end{array}$ \\
\hline \multirow{6}{*}{ 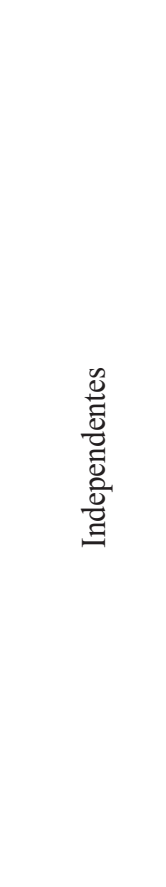 } & 2. Ambiente & Cerro Negro (2005) & Concórdia (2013) & $\begin{array}{c}\text { São Pedro de } \\
\text { Alcântara (2010) } \\
\text { Rio Negrinho (2010) } \\
\text { Herval d'Oeste (2013) }\end{array}$ \\
\hline & 3a. Idade do Prefeito & Mafra (2012) & $\begin{array}{l}\text { Barra Velha } \\
\quad(2011)\end{array}$ & $\begin{array}{c}\text { Belmonte (2016) } \\
\text { Benedito Novo (2005) }\end{array}$ \\
\hline & $\begin{array}{l}\text { 3b. Escolaridade do } \\
\text { Prefeito }\end{array}$ & $\begin{array}{l}\text { Bombinhas (2009-2012) } \\
\text { Luiz Alves (2005-2008) } \\
\text { Rio das Antas (2013-2016) } \\
\text { Romelândia (2009-2012) } \\
\text { Timbó Grande (2005-2008) }\end{array}$ & $\begin{array}{c}\text { Biguaçu } \\
(2005-2008)\end{array}$ & $\begin{array}{l}48 \text { municípios } \\
(2005-2016)\end{array}$ \\
\hline & $\begin{array}{l}\text { 4. Despesa de Pessoal } \\
\text { per capita }\end{array}$ & Criciúma (2006) & $\begin{array}{l}\text { Bom Retiro } \\
\quad(2016)\end{array}$ & $\begin{array}{c}\text { Nova Itaberaba }(2010) \\
\text { Laurentino }(2012) \\
\text { Taió }(2013)\end{array}$ \\
\hline & $\begin{array}{l}\text { 5a. Densidade } \\
\text { Populacional }\end{array}$ & Capão Alto (2005-2016) & $\begin{array}{l}\text { Balneário } \\
\text { Camboriú } \\
(2005-2016)\end{array}$ & $\begin{array}{l}\text { Balneário Barra do } \\
\text { Sul (2013) } \\
\text { Rodeio }(2010) \\
\text { Laurentino }(2016)\end{array}$ \\
\hline & $\begin{array}{l}\text { 5b. Logarítimo da } \\
\text { População }\end{array}$ & Santiago do Sul (2009-2016) & $\begin{array}{c}\text { Joinville } \\
(2005-2016)\end{array}$ & $\begin{array}{c}\text { Saudades (2008) } \\
\text { Santa Terezinha } \\
(2013) \\
\text { Lontras }(2005)\end{array}$ \\
\hline
\end{tabular}

Fonte:elaborada pelos autores.

O critério para a inclusão do município na coluna média foi ele apresentar um resultado mais próximo da média da variável apontado na estatística descritiva, adicionado o município anterior e posterior mais próximo a média. Assim, exceto as variáveis Escolaridade do Prefeito e Idade do Prefeito, as demais compreendem três municípios. Exceto essas variáveis por apresentarem escala numérica cardinal, não comportando valores decimais. 
Ainda sobre a variável Idade do Prefeito, o município com o prefeito de menor idade a assumir mandato eleitoral nas últimas três eleições catarinenses foi Mafra, com 24 anos. Em contraponto, Barra Velha, no ano de 2011, teve o prefeito de maior idade. Porém o prefeito de maior idade teve seu mandado interrompido em 2011 (terminaria em 2012) por impeachment.

A variável indicadora do desempenho municipal apresenta Brunópolis, no ano de 2010, como o município com pior desempenho e o município de Bom Retiro, no ano de 2016, com o melhor desempenho. O município de Bom Retiro (ano 2016), além de apresentar maior desempenho, alcançou a marca da maior despesa de pessoal per capita da amostra. A menor Despesa de Pessoal per capita foi identificada no município de Criciúma (2006). O município de Cerro Negro, no ano de 2005, apresentou maior incerteza ambiental, com o menor Índice de Desenvolvimento Humano Municipal da amostra. Em contraponto, Concórdia, no ano de 2013, apresentou menor incerteza ambiental.

O município de Capão do Alto (ano 2016) possui a menor densidade populacional. Ao longo dos 12 anos, o município se mantém, alternando os anos, mas sempre com menor densidade populacional. Já o município com maior densidade populacional é Balneário Camboriú (ano 2016), e conforme os dados coletados de 2005 a 2016 fixa-se como o município com maior densidade populacional.

Face à evidência na estatística descritiva do município de Bom Retiro possuir maior Receita Corrente Líquida per capita e maior Despesa de Pessoal per capita, é relevante analisar a matriz de correlação entre as variáveis do estudo para verificar a tendência de alinhamento ou não das variáveis. Na Tabela 4 apresenta-se a matriz de correlação entre as variáveis.

Observa-se correlação forte, significante e positiva entre as variáveis Receita Corrente Líquida per capita e Despesa com Pessoal per capita. Isso sugere que quanto maior a receita corrente líquida do município maior será a despesa com pessoal, pois a Lei Complementar $n^{\circ} 101 / 2000$ estabelece para os municípios que a despesa de pessoal não pode ser maior que $60 \%$ da receita corrente líquida. A estatística descritiva reforça que o município com maior Receita Corrente Líquida per capita também possui maior Despesa com Pessoal per capita. Porém, há outros elementos envolvidos, pois o contrário não é verdadeiro. A estatística descritiva mostra que o município com menor Receita Corrente Líquida per capita não é o que apresenta menor Despesa com Pessoal per capita. Há especificidades dos municípios que impactam a relação das variáveis contingenciais com o desempenho.

Ainda com base na variável Receita Corrente Líquida per capita, nota-se uma correlação moderada, negativa e significativa com a variável população, ou seja, quanto maior a população menor tende a ser o desempenho municipal. Este achado alinha-se às perspectivas teóricas de small is beautifulI (Schumacher, 1973), de que municípios menores possuem melhor desempenho. E uma correlação moderada, positiva e significativa com o Produto Interno Bruto per capita.

A variável ambiente apresenta correlação moderada, positiva e significativa com as Densidade Populacional, Logaritmo da População e Produto Interno Bruto per capita. Pode-se atribuir esta correlação à composição da variável Índice de Desenvolvimento Humano Municipal, que nesta pesquisa representa a variável contingencial externa, por compreender dados da população referente a emprego, renda, saúde e educação.

Correlação moderada, negativa e significativa é observada entre Despesa com Pessoal per capita e Logaritmo da População. Isso indica que as despesas com pessoal dos municípios não acompanham o aumento populacional. Este achado pode auxiliar na explicação do déficit de atendimento em saúde, educação e segurança, mas merece mais pesquisas futuras. 
Tabela 4. Matriz de correlação

\begin{tabular}{|c|c|c|c|c|c|c|c|c|c|c|c|}
\hline Variáveis & 1. RCLpc & 2. Amb & 3a. IP & 3b. EP & 4. DPpc & 5a. DensP & 5b. LP & 6. AP & 7. ExP & 8. DirP & 9. PIBpc \\
\hline 1. RCLpc & 1 & & & & & & & & & & \\
\hline 2. Amb & $0,104 *$ & 1 & & & & & & & & & \\
\hline 3a. IP & $0,039 *$ & $0,079^{*}$ & 1 & & & & & & & & \\
\hline 3b. EP & $-0,024$ & $0,207^{*}$ & $-0,174$ & 1 & & & & & & & \\
\hline 4. DPpc & $0,961^{*}$ & $0,120^{*}$ & $0,067^{*}$ & $-0,017$ & 1 & & & & & & \\
\hline 5a. DensP & $-0,048^{*}$ & $0,345^{*}$ & $-0,025$ & $0,145^{*}$ & $-0,038$ & 1 & & & & & \\
\hline 5b. LP & $-0,424 *$ & $0,465^{*}$ & $0,067^{*}$ & $0,258^{*}$ & $-0,359^{*}$ & $0,546^{*}$ & 1 & & & & \\
\hline 6. AP & $0,149^{*}$ & $0,131^{*}$ & 0,007 & $0,036^{* *}$ & $0,174 *$ & 0,030 & $0,041 * *$ & 1 & & & \\
\hline 7. ExP & $-0,018$ & $-0,009$ & $-0,004 *$ & $0,004^{*}$ & 0,011 & 0,012 & 0,013 & 0,020 & 1 & & \\
\hline 8. DirP & $0,056^{*}$ & 0,021 & 0,108 & $-0,019$ & $0,062 *$ & $0,039 * *$ & 0,004 & $-0,423^{*}$ & $-0,043 * *$ & 1 & \\
\hline 9. PIBpc & $0,387^{*}$ & $0,475^{*}$ & $0,106^{*}$ & $0,139 *$ & $0,395^{*}$ & $0,146^{*}$ & $0,207^{*}$ & $0,137^{*}$ & $-0,025$ & $0,045^{*}$ & 1 \\
\hline
\end{tabular}

Fonte:elaborada pelos autores.

Legenda: $* * \mathrm{p}<0,05 ; * \mathrm{p}<0,01$ 
Na Tabela 5 apresentam-se os resultados do modelo que testa a influência dos fatores contingenciais no desempenho municipal.

Tabela 5. Regressão de dados em painel

\begin{tabular}{|c|c|c|c|c|}
\hline Receita Corrente Líquida per capita & $\beta$ & Estatística t & p-valor & $e$ \\
\hline \multicolumn{5}{|c|}{ Variável contingencial externa } \\
\hline 2. Ambiente & 421,2761 & 3,79 & 0,000 & 111,2273 \\
\hline \multicolumn{5}{|c|}{ Variáveis contingenciais internas } \\
\hline \multicolumn{5}{|l|}{ Liderança organizacional: } \\
\hline 3a. Idade do Prefeito & 1,9980 & 2,64 & 0,008 & 0,7566 \\
\hline 3b. Escolaridade do Prefeito & 2,2978 & 0,68 & 0,497 & 3,3861 \\
\hline \multicolumn{5}{|l|}{ Estrutura: } \\
\hline 4. Despesa de Pessoal per capita & 1,6599 & 63,61 & 0,000 & 0,0261 \\
\hline \multicolumn{5}{|l|}{ Porte organizacional: } \\
\hline 5a. Densidade Populacional & 0,3552 & 4,05 & 0,000 & 0,0876 \\
\hline 5b. Logarítimo da População & $-205,0721$ & $-13,11$ & 0,000 & 15,6413 \\
\hline \multicolumn{5}{|c|}{ Variáveis de controle } \\
\hline 6. Alinhamento Partidário & 23,1981 & 2,51 & 0,012 & 9,2363 \\
\hline 7. Ex-Prefeito & $-4,3935$ & $-0,46$ & 0,647 & 9,6076 \\
\hline 8. Direção Partidária & $-0,4058$ & $-0,03$ & 0,976 & 13,4226 \\
\hline 9. Produto Interno Bruto per capita & 2,8962 & 2,10 & 0,036 & 1,3792 \\
\hline \multicolumn{5}{|c|}{ Regressão linear } \\
\hline Constante & $1.765,22$ & 12,46 & 0,000 & 141,6499 \\
\hline Número de observações & \multicolumn{4}{|c|}{3.516} \\
\hline $\mathrm{F}$ & \multicolumn{4}{|c|}{585,24} \\
\hline Prob. $>\mathrm{F}$ & \multicolumn{4}{|c|}{0,0000} \\
\hline $\mathrm{R}^{2}$ & \multicolumn{4}{|c|}{0,7795} \\
\hline Durdin-Watson & \multicolumn{4}{|c|}{0,4428} \\
\hline
\end{tabular}

Fonte:elaborada pelos autores.

Observa-se que as variáveis independentes e de controle explicam em $77,95 \%\left(\mathrm{R}^{2}=0,7795\right)$ a variável dependente desempenho municipal. Recomenda-se, assim, que estudos futuros incluam outras variáveis para analisar o desempenho municipal. Posto esta limitação do estudo, ressalta-se a adequação da estimação do modelo ao utilizar o mínimos quadrados generalizados factíveis com autocorrelação controlada por Durbin-Watson e estimativa robusta do erro (Prob. $>\mathrm{F}=0,0000$ e DW=0,4428) (Gujarati \& Porter, 2011; Fávero \& Belfiore, 2017).

Portanto, as variáveis contingenciais externa e internas influenciam significativamente o desempenho municipal. Essa inferência baliza-se na influência positiva e significativa encontrada nas variáveis independentes ambiente, idade do prefeito, despesa de pessoal per capita e densidade populacional, bem como na influência negativa e significativa da varável população. Nota-se, também, influência positiva e significativa das variáveis de controle alinhamento partidário e Produto Interno Bruto per capita no desempenho municipal.

Em consonância com a classificação proposta por Wadongo e Abdel-Kader (2014), para os fatores contingenciais analisados nos municípios catarinenses, tem-se influência de fatores contingenciais internos (liderança organizacional, estrutura e porte organizacional) e externo (ambiente). Portanto, corrobora-se com Teisman e Klijn (2008), que as organizações absorvem pressões externas e internas e isso impacta sua estrutura e desempenho.

Com estes resultados chama-se atenção das pesquisas que utilizam a nova gestão pública como base teórica, visto que seu foco está na implantação de sistemas de controle gerencial, prestação de contas, transparência e desempenho (Woods, 2009). Argumenta-se que é necessário observarem os fatores contingenciais relacionadas ao desempenho e mudanças organizacionais. Christensen e Yoshimi (2003) salientam que a Nova Gestão Pública não explica completamente o desempenho, logo, pesquisadores deveriam usar outras lentes para auxiliar na explicação do desempenho, por exemplo, a Teoria Contingencial. 
Os achados deste estudo corroboram os de Christensen e Yoshimi (2003), pois foi observada influência de fatores contingenciais no desempenho. Wadongo e Abdel-Kader (2014) destacam que a variável ambiente é um fator contingencial relevante pela incerteza ou dificuldade de previsão. Os resultados revelam que nos municípios catarinenses, nos anos analisados, o ambiente influenciou positivamente o desempenho municipal, assim, quanto maior for o Índice de Desenvolvimento Humano dos municípios maior será seu desempenho.

Outra variável com influência positiva e significativa é a liderança organizacional, capturada pela idade do prefeito. Este achado diverge de Avellaneda e Gomes (2015) e corrobora com George, Van de Valle e Hammerschmid (2019), por evidenciar que as características do prefeito influenciam o desempenho da organização. A variável escolaridade do prefeito, que compunha o fator contingencial da liderança organizacional, não influenciou significativamente o desempenho, o que diverge da pesquisa de Avellaneda e Gomes (2015).

Além das variáveis de liderança organizacional, atende-se ao chamado de Greenwood \& Hinings (1976), por verificar a influência de características do representante eleito. A variável de controle alinhamento partidário influenciou positiva e significativamente o desempenho. Nos municípios em que o partido do prefeito é o mesmo do governador há maior possibilidade de obtenção de recursos, aumentando a Receita Corrente Líquida per capita. As demais variáveis de controle atreladas às características do prefeito não obtiveram significância estatística. Assim, a reeleição do prefeito e sua direção partidária não influenciaram no desempenho municipal.

Observa-se ainda que as variáveis contingenciais estrutura e porte organizacional influenciaram positivamente o desempenho. Tem-se que quanto maior a despesa de pessoal e a densidade populacional maior será o desempenho municipal. A relação positiva e significativa entre estrutura e desempenho municipal indica que o corte de gastos com pessoal nos municípios estudados não aumentará seu desempenho. Ressalta-se que quanto maior o desempenho (Receita Corrente Líquida) mais recursos o município terá para gerir. Isso instiga pesquisas futuras relacionar Despesa de Pessoal per capita com efetividade e eficiência na prestação de serviços públicos.

A relação positiva e significativa entre densidade populacional e desempenho municipal sugere que a urbanização dos municípios contribui para o aumento da Receita Corrente Líquida. Assim, estudos futuros podem analisar a influência de políticas públicas de urbanização no desempenho municipal. Varela, Martins e Fávero (2012) pesquisaram a área da saúde de municípios de São Paulo e constataram que a urbanização dos municípios reduz as despesas aplicadas à saúde, mas aumenta o desempenho municipal.

A variável população, que integra o fator contingencial porte organizacional, exerceu influência significativa e negativa no desempenho. Assim, quanto maior a população menor o desempenho municipal. Este achado diverge do estudo de Avellaneda e Gomes (2015), que encontraram influência positiva entre a população e o desempenho dos municípios de Minas Gerais nos anos de 2005 a 2007.

Por fim, importa destacar a evidência inferencial que os fatores contingenciais internos e externo influenciam o desempenho municipal. Desse modo, ressalvadas as especificidades apontadas por Woods (2009), o setor público alinha-se às perspectivas do setor privado (Beuren \& Fiorentin, 2014; Fagundes et al., 2010) e terceiro setor (Oliveira \& Callado, 2018), pela influência dos fatores contingenciais no desempenho.

\section{CONSIDERAÇÕES FINAIS}

Este estudo analisou a influência dos fatores contingenciais no desempenho de municípios ao longo de três mandatos governamentais. Os resultados da análise de 293 municípios revelaram que os fatores contingenciais ambiente, liderança organizacional, estrutura e porte organizacional impactaram significativamente o desempenho municipal. As variáveis Índice de Desenvolvimento Humano Municipal (ambiente), idade do prefeito (liderança organizacional), despesa de pessoal per capita (estrutura) e densidade populacional (porte organizacional) influenciaram positivamente o desempenho municipal. Já a variável população (porte organizacional) influenciou negativamente o desempenho municipal.

Quanto às variáveis de controle, os resultados demonstraram que o alinhamento partidário entre prefeito e governador e o Produto Interno Bruto per capita influenciaram positiva e significativamente o desempenho municipal. A variável escolaridade do prefeito, do construto liderança organizacional, e as variáveis de controle reeleição (ex-prefeito) e direção partidária (direita, esquerda ou centro) não foram estatisticamente significativas.

Este estudo avança na literatura relativa à Teoria Contingencial e análise do desempenho no setor público, em especial por considerar um período de análise longitudinal e privilegiar uma abordagem quantitativa. Contribui ainda por evidenciar indicadores que podem ser utilizados para capturar o desempenho municipal e os fatores contingenciais ao longo do tempo, dado o desafio em pesquisas com indicadores no setor público quanto à disponibilidade de dados de municípios. 
Na perspectiva teórica, chama-se atenção da literatura dedicada à Nova Gestão Pública (New Public Management) ao demonstrar que fatores contingenciais influenciam (positiva e negativamente) o desempenho municipal. Nesta perspectiva, destaca-se a pesquisa de Christensen e Yoshimi (2003), que evidencia que a nova gestão pública não explica sozinha o desempenho e indica a análise da influência do desempenho sob lentes diversas, dentre elas a Teoria Contingencial.

O estudo também apresenta contribuições práticas à gestão pública no que concerne aos fatores contingenciais que afetam a estrutura, o comportamento organizacional (pela revisão da literatura) e o desempenho de municípios (na análise dos dados). Assim, pode-se buscar o alinhamento entre o ambiente e a estrutura com vistas a maximizar o desempenho e a eficiência da organização (Brignall \& Modell, 2000). O estudo contribui com elementos que devem ser considerados na elaboração de políticas públicas e ações governamentais que visam melhor desempenho dos municípios.

Como limitações da pesquisa destaca-se a escolha teórica das variáveis utilizadas para capturar o desempenho e os fatores contingenciais, além das variáveis de controle. Como o modelo explica a variável independente em 77,95\%, variáveis não incluídas influenciam o desempenho municipal. Assim, estudos futuros podem adicionar ao modelo tecnologia, estratégia (Beuren \& Fiorentin, 2014), estrutura mecânica e orgânica (Fagundes et al., 2010), práticas gerenciais (Gonzaga et al., 2016) e cultura organizacional (Oliveira \& Callado, 2018).

O contexto da pesquisa, circunscrito aos municípios de Santa Catarina, também é uma limitação. Fatores contingenciais internos e externos podem estar sob influência da localização, cultura e especificidades do Estado. Outra limitação refere-se à escala utilizada para a variável capacidade do prefeito frente à escolaridade e direção partidária, por estar parametrizada em escala ordinal, visto caracterizar distância direta entre as escolaridades e as direções partidárias. A pesquisa limita-se também por utilizar a definição de Carreirão (2006) para direção partidária, que pode ter-se alterado no lapso temporal pesquisado.

\section{REFERÊNCIAS}

Adhikari, A., Derashid, C., \& Zhang, H. (2006). Public policy, political connections, and effective tax rates: Longitudinal evidence from Malaysia. Journal of Accounting and Public Policy, 25(5), 574-595. DOI: https:// doi.org/10.1016/j.jaccpubpol.2006.07.001.

Avellaneda, C.N., \& Gomes, R.C. (2015). Is small beautiful? Testing the direct and nonlinear effects of size on municipal performance. Public Administration Review, 75(1), 137-149. DOI: https://doi.org/10.1111/ puar.12307.

Avellaneda, C.N., \& Gomes, R.C. (2017). Mayoral quality and municipal performance in Brazilian local governments. Organizações \& Sociedade, 24(83), 555-579. DOI: http://dx.doi.org/10.1590/1984-9240831.

Beuren, I.M., \& Fiorentin, M. (2014). Influência de Fatores Contingenciais nos Atributos do Sistema de Contabilidade Gerencial: um estudo em empresas têxteis do Estado do Rio Grande do Sul. Revista de Ciências da Administração, 16(38), 196-212. DOI: https://doi.org/10.5007/2175-8077.2014v16n38p195.

Bezerra, V.V.N., \& Carvalho, J.R.M. (2018). Avaliação de desempenho por funções de governo através do método PROMETHEE II. Contextus. Revista Contemporânea de Economia e Gestão, 16(3), 40-65. DOI: https://doi. org/10.19094/contextus.v16i3.39904.

Boyne, G.A., Meier, K.J., O'Toole, L.J, \& Walker, R.M. Where next? Research directions on performance in public organizations. Journal of Public Administration Research and Theory, 15(4), 633-639. DOI: https:// doi.org/10.1093/jopart/mui037.

Brignall, S., \& Modell, S. (2000). An institutional perspective on performance measurement and management in the 'new public sector'. Management Accounting Research, 11(3), 281-306. DOI: https://doi.org/10.1006/ mare.2000.0136.

Castaldelli, E., Jr., \& de Aquino, A.C.B. (2011). Indicadores de desempenho em entidades fiscalizadoras superiores: o caso brasileiro. Contabilidade Vista \& Revista, 22(3),15-40.

Carreirão, Y.S. (2006). Ideologia e partidos políticos: um estudo sobre coligações em Santa Catarina. Opinião Pública, 12(1), 136-163. DOI: http://dx.doi.org/10.1590/S0104-62762006000100006.

Christensen, M., \& Yoshimi, H. (2003). Public sector performance reporting: new public management and contingency theory insights. Government Auditing Review, 10(3), 71-83. 
Donaldson, L. (2001). The contingency theory of organizations. Thousand Oaks: Sage.

Drew, J., Kortt, M.A., \& Dollery, B. (2014). Economies of scale and local government expenditure: evidence from Australia. Administration \& Society, 46(6), 632-653. DOI: https://doi.org/10.1177/0095399712469191.

Fagundes, J.A., Petri, M., Lavarda, R.B., Rodrigues, M.R., Lavarda, C.E.F., \& Soller, C.C. (2011). Estrutura organizacional e gestão sob a ótica da teoria da contingência. Gestão \& Regionalidade, 26(78), 52-63. DOI: https://doi.org/10.13037/gr.vol26n78.792.

Fávero, L.P., \& Belfiore, P. (2017). Manual de análise de dados: estatística e modelagem multivariada com Excel, SPSS e Stata. Rio de Janeiro: Elsevier.

George, B., Van de Walle, S., \& Hammerschmid, G. (2019). Institutions or Contingencies? A Cross-Country Analysis of Management Tool Use by Public Sector Executives. Public Administration Review, 79(3), 330342. DOI: https://doi.org/10.1111/puar.13018.

Goeminne, S., \& George, B. (2019). New development: Determinants of financial performance in public organizations. Public Money \& Management, 39(1), 70-73. DOI: https://doi.org/10.1080/09540962.2018.1 476309.

Gonzaga, R.P., Frezatti, F., Ckagnazaroff, I.B., \& Suzart, J.A.S. (2016). Avaliação de desempenho no governo mineiro: alterações dos indicadores e metas. Revista de Administração Contemporânea, 21(spec.), 1-21. DOI: http://dx.doi.org/10.1590/1982-7849rac2017150331.

Gujarati, D.N., \& Porter, D.C. (2011). Econometria básica (5. ed.). São Paulo: McGraw Hill.

Greenwood, R., \& Hinings, C.R. (1976). Contingency theory and public bureaucracies. Policy \& Politics, 5(2), 159-180. DOI: https://doi.org/10.1332/030557376782842986.

Hedeker, D., \& Gibbons, R.D. (2006). Longitudinal data analysis (Vol. 451). New Jersey: John Wiley \& Sons.

Holzer, M., Fry, J., Charbonneau, E., Van Ryzin, G., Wang, T., \& Burnash, E. (2009). Literature review and analysis related to optimal municipal size and efficiency. Rutgers-Newark: School of Public Affairs and Administration.

Hood, C. (1991). A public management for all seasons? Public Administration, 69(1), 3-19. DOI: https://doi. org/10.1111/j.1467-9299.1991.tb00779.x.

Johnsen, A. (2005). What does 25 years of experience tell us about the state of performance measurement in public policy and management? Public Money and Management, 25(1), 9-17. DOI: https://doi.org/10.1111/j.14679302.2005.00445.x.

Jung, C.S., \& Kim, S.E. (2014). Structure and perceived performance in public organizations. Public Management Review, 16(5), 620-642. DOI: https://doi.org/10.1080/14719037.2012.743576.

Lapsley, I. (1999). Accounting and the new public management: instruments of substantive efficiency or a rationalising modernity? Financial Accountability \& Management, 15(3-4), 201-207. DOI: https://doi. org/10.1111/1468-0408.00081.

Lei Complementar $n^{\circ}$. 101, de 4 de março de 2000. Estabelece normas de finanças públicas voltadas para a responsabilidade na gestão fiscal e dá outras providências. Recuperado de http://www.planalto.gov.br/ ccivil_03/leis/lcp/lcp101.htm

Lima, D.V.D., \& Aquino, A.C.B.D. (2019). Resiliência financeira de fundos de regimes próprios de previdência em municípios. Revista Contabilidade \& Finanças, 30(81), 425-445. DOI: http://dx.doi.org/10.1590/1808$057 \times 201908810$.

Marques, K.C.M., Souza, R.P. \& Silva, M.Z. (2015). Análise de SWOT da abordagem da contingência nos estudos da contabilidade gerencial. Revista Contemporânea de Contabilidade, 12(25), 117-136. DOI: http://dx.doi. org/10.5007/2175-8069.2015v12n25p117.

Oliveira, A.S., \& Callado, A.A.C. (2018). Fatores contingenciais e o controle gerencial: uma avaliação em organizações não governamentais (ONGS) brasileiras. Advances in Scientific and Applied Accounting, 11(1), 092-109. DOI: http://dx.doi.org/10.14392/ASAA.2018110105.

Oliveira, F.B.D., Sant'Anna, A.D.S., \& Vaz, S.L. (2010). Liderança no contexto da nova administração pública: uma análise sob a perspectiva de gestores públicos de Minas Gerais e Rio de Janeiro. Revista de Administração Pública, 44(6), 1453-1475. DOI: https://doi.org/10.1590/S0034-76122010000600009. 
Park, S. (2019). Size matters: Toward a Contingency Theory of diversity effects on performance. Public Performance \& Management Review. DOI: http://dx.doi.org/10.1080/15309576.2019.1657917.

Parker, L. \& Gould, G. (1999). Changing public sector accountability: Critiquing new directions. Accounting Forum, 23 (2), 109-135. DOI: http://dx.doi.org/10.1111/1467-6303.00007.

Poi, B., \& Wiggins, V. (2001). Testing for panel-level heteroskedasticity and autocorrelation. StataCorp LP.

Porporato, M. (2011). Management Control Systems' literature Development: Theoretical Approaches and Critiques Within the Functionalist Paradigm. Revista Universo Contábil, 7(2), 146-173. DOI: http://dx.doi. org/10.4270/ruc.20117.

Ramos, M.P., Schabbach, L.M. (2012). O estado da arte da avaliação de políticas públicas: conceituação e exemplos de avaliação no Brasil. Revista de Administração Pública, 46(5), 1271-1294.

Schumacher, E.F. (1973). Small is beautiful: A study of ecomonics as if people mattered. New York: Vintage.

Teisman, G.R. \& Klijn, E. (2008): Complexity Theory and Public Management. Public Management Review, 10(3), 287-297. DOI: https://doi.org/10.1080/14719030802002675.

Tribunal de Contas da União (TCU). Acórdão 825/2015, de 15 de abril de 2015. Atrasos nos repasses a Bancos Federais. Recuperado abril 08, 2020, de file://C:/Users/Ilse/Downloads/Atrasos\%20nos\%20repasses\%20 a\%20bancos\%20federais_WEB.pdf

Van der Hoek, M.P. (2005). From cash to accrual budgeting and accounting in the public sector: The Dutch experience. Public Budgeting \& Finance, 25(1), 32-45. DOI: https://doi.org/10.1111/j.0275-1100.2005.00353.x.

Van de Walle, S., \& Hammerschmid, G. (2011). The impact of the new public management: Challenges for coordination and cohesion in European public sectors (review essay). Halduskultuur - Administrative Culture, 12(2), 190-209.

Varela, P.S., Martins, G.A, \& Fávero, L.P.L. (2012). Desempenho dos municípios paulistas: uma avaliação de eficiência da atenção básica à saúde. Revista de Administração, 47(4), 624-637. DOI: https://doi.org/10.5700/ rausp1063.

Verbeeten, F.H. (2008). Performance management practices in public sector organizations: Impact on performance. Accounting, Auditing \& Accountability Journal, 21(3), 427-454. DOI: https://doi. org/10.1108/09513570810863996.

Wadongo, B., \& Abdel-Kader, M. (2014). Contingency theory, performance management and organisational effectiveness in the third sector: A theoretical framework. International Journal of Productivity and Performance Management, 63(6), 680-703. DOI: https://doi.org/10.1108/IJPPM-09-2013-016.

Woods, M. (2009). A contingency theory perspective on the risk management control system within Birmingham City Council. Management Accounting Research, 20(1), 69-81. DOI: https://doi.org/10.1016/j.mar.2008.10.003.

\section{Como citar este artigo}

Sell, F. F., Beuren, I. M., \& Lavarda, C. E. F. (2020). Influência de fatores contingenciais no desempenho municipal: evidências inferenciais. Revista de Contabilidade e Organizações, 14:e164058. DOI: http:// dx.doi.org/10.11606/issn.1982-6486.rco.2020.164058 